\title{
Indoor WLAN Localization using Group Sparsity Optimization Technique
}

\author{
Ali Khalajmehrabadi, Nikolaos Gatsis, and David Akopian \\ Department of Electrical and Computer Engineering, University of Texas at San Antonio \\ One UTSA Circle, San Antonio, Texas 78249-0669, USA
}

\begin{abstract}
This paper proposes a novel approach for Wireless Local Area Networks (WLAN) indoor fingerprinting localization using a novel interpretation and optimization solution. The user's location is introduced as a sparse vector which can be estimated in a single minimization problem. The coarse localization is embedded in the fine localization to prevent the cases where the user's position is searched in wrong subset of reference points (RPs). First, the RPs are clustered in layers using the similarity between the online measurement and RP fingerprints via an AP coverage vector. Then, the localization approach is performed via a joint minimization of convex least squares minimization of residuals, $\ell_{1}$-norm of the entire position vector, and weighted $\ell_{2}$ norm of groups of RPs. The method has been evaluated though Monte Carlo simulation runs and the results showed a great positioning accuracy.
\end{abstract}

\section{INTRODUCTION}

Indoor localization based on Wireless Local Area Networks (WLAN) Received Signal Strength (RSS) fingerprints is a promising state-of-the-art approach that exploits the so-called radio map surveying of wireless signal characteristics from available Access Points (APs), called fingerprints, on a grid of locations, otherwise referred to as a grid of anchor points or reference points (RPs). The RSS measurements are commonly used to characterize wireless signals. They are collected offline during surveying and associated data pairs (location, fingerprint) for all anchor points are saved in the radio map database. Actual positioning of the users, also referred to as online phase of WLAN fingerprinting techniques, captures signal fingerprints on the current user location and associates it with the most likely fingerprint entries of the radio map. In other words, the task of a fingerprint positioning system is to estimate the position of the user through a comparison mechanism between the online measurement and radio map. This problem has been previously addressed from various perspectives including statistical and optimization techniques [1]-[9].

There are several challenges associated with WLAN fingerprinting localization. The RSS fingerprints fluctuates randomly in a location which prevents establishing a distance-location relation with the APs. These fluctuations come from the RSS multipath profile due to the presence of a non-line-of-sight (NLOS) propagation in indoor environments with obstacles such as furnitures, doors, walls, etc. [8], [9]. In addition, RSS profiles have shown to be highly varying in close distances to APs [10].
The fingerprinting localization approaches are divided in two broad categories: deterministic methods [11] which use a specific distance metric between the online and radio map fingerprints; and probabilistic methods [12] which estimate the user's location based on maximum statistical likelihood (ML).

In general, a typical fingerprinting positioning consists of three steps: 1) coarse localization, i.e. the selection of a subset of RPs where most probably the user exist; 2) selection of APs as using the whole set of APs make the localization biased, time-consuming, and computationally intensive; 3 ) fine localization where the fine location of the user is estimated through a rule that associates online measurement.

One of the recent probabilistic approaches has benefited from kernel functions [2]. This approach constrains the localization region to a subset of RPs using an AP coverage vector that compares the difference between the online measurements and radio map. They select APs from a subset of strongest APs, Bhattacharyya distance, and Information Potential. Kernel functions play the role of estimating the RSS probability distribution function and are used to find the ML of user's location.

In particular, the localization problem can be considered as solving a linear system of equations in which the coefficient matrix is the radio map, the observation vector is the user's online RSS measurement, and the position vector is the unknown so-called user's location vector to be estimated [5] and [6]. Since the user's position is a single point among all available RPs, the unknown position vector is considered as a sparse vector. Due to the sparsity of the position vector, the solution of the system can be obtained with an $\ell_{1}$-norm minimization, called compressive sensing (CS), rather than a simple pseudo-inverse solution. While $\ell_{1}$-norm solution has been proposed in the state-of-the-art, however, it does not consider minimization of residuals as another indication of location accuracy. Also, a certain type of orthogonalization on the radio map is needed to obtain a unique solution in the $\ell_{1}$ norm minimization algorithm. In addition, the previous studies have to use pre-processing coarse localization approaches to scale down the complexity of the solution to a smaller subregion solution. The coarse localization is prone to errors and may need manual arrangements.

This paper proposes a novel WLAN localization approach so that the user's location can be estimated in a single minimization problem, which can be solved quickly through available solvers. Both coarse and fine localization are com- 
bined in a single optimization problem so that the need for a pre-coarse localization step is eliminated. The minimization does not confine the problem to a specific subset of RPs as all points have a weight to participate in localization. This reduces the coarse localization errors in cases where the user's position is estimated in wrong subset of RPs, which leads to high positioning error. In the proposed approach, at first, we compute the similarity between the online measurement and that of each RP via an AP coverage vector. Through this similarity, the RPs are clustered in layered groups. Then, we compute the weights for each group of RPs that is proportional to the inverse of the group Hamming distance. Our approach selects the APs based on the Fisher criterion score assigned to each AP as it provides a good measure of the maximum differentiability of each AP over RPs.

In this paper, the CS WLAN localization definitions and formulation is elaborated in Section II. A simple online RP clustering method is introduced in Section III, and the localization procedure is described in Section IV. Section V illustrates the simulation results followed by conclusions in Section VI.

\section{WLAN LOCALIZATION: DEFINITION AND FORMULATION}

In this section, we first provide the fundamentals of WLAN fingerprinting localization followed by the equivalent CS problem formulation. The area of interest is placed in a set of RPs $\mathcal{P}=\left\{\mathbf{p}_{j}=\left(x_{j}, y_{j}\right) \mid \forall j=1, \cdots, N\right\}$ where $\mathcal{P}$ defines the set of RP Cartesian coordinates. Without loss of generality the analysis are provided for a two dimensional space. At each RP, the RSS fingerprints are recorded at time instants $t_{m}, m=1, \ldots, M$ and are denoted by $r_{j}^{i}\left(t_{1}\right), \cdots, r_{j}^{i}\left(t_{M}\right)$, where $i$ indicates the AP index from the set of APs $\mathcal{A}=\left\{A P^{1}, \ldots, A P^{L}\right\}$. The RSS fingerprints from all APs at $\mathbf{p}_{j}$ and at time $t_{m}$ are organized in a vector $\mathbf{r}_{j}\left(t_{m}\right)=\left[r_{j}^{1}\left(t_{m}\right), \cdots, r_{j}^{L}\left(t_{m}\right)\right]^{T}$. Organizing these vectors for all fingerprinting instants constructs a $L \times N \times M$ matrix called radio map. The time-averaged radio map can be represented as

$$
\boldsymbol{\Psi}=\left(\boldsymbol{\psi}_{1}, \cdots, \boldsymbol{\psi}_{N}\right)=\left(\begin{array}{ccc}
\psi_{1}^{1} & \cdots & \psi_{N}^{1} \\
\vdots & \ddots & \vdots \\
\psi_{1}^{L} & \cdots & \psi_{N}^{L}
\end{array}\right)
$$

where $\boldsymbol{\psi}_{j}=\left[\psi_{j}^{1}, \ldots, \psi_{j}^{L}\right]^{T}$, and $\psi_{j}^{i}=\frac{1}{M} \sum_{m=1}^{M} r_{j}^{i}\left(t_{m}\right)$. In general, $\psi_{j}^{i}$ is a representative fingerprint measurement and can be selected using alternative ways as well.

The mobile user observes the online measurements, $\boldsymbol{y}=$ $\left(y^{1}, y^{2}, \ldots, y^{L}\right)$. The objective of localization scheme is to estimate the mobile user's location, $\hat{\mathbf{p}}=(\hat{x}, \hat{y})$, using the received online vector and the radio map.

The premise of sparsity-based approaches in localization is that the location of the user can be approximately represented as a 1 -sparse location indicator vector $\boldsymbol{\theta}=[0, \ldots, 0,1,0, \ldots, 0]^{T}$ where each entry corresponds to one RP and 1 shows the index of the RP that is closest to the user's location.

\section{GROUP SPARSITY-BASED LOCALIZATION}

As described in Section II, the user's location can be defined as a sparse vector whose single entry equal to 1 corresponds to the RP that is closest to the user. The sparse recovery algorithms have mainly concentrated on recovering a sparse position matrix. The CS WLAN localization needs a preprocessing step for online coarse localization, in order to reduce the complexity of searching over all available RPs.

However, there is no guarantee that the cluster within which the solution is searched is the best cluster especially when the clusters are disjoint. Our proposition is to utilize all the clusters, each with a different share. To this end, we propose the group sparsity-based localization in which a sparse user's position vector is recovered by jointly searching all clusters

$$
\hat{\boldsymbol{\theta}}=\underset{\boldsymbol{\theta}}{\operatorname{argmin}}\left[\frac{1}{2}\|\mathbf{y}-\boldsymbol{\Phi} \boldsymbol{\Psi} \boldsymbol{\theta}\|_{2}^{2}+\lambda_{1}\|\boldsymbol{\theta}\|_{1}+\lambda_{2} \sum_{k=1}^{K} w_{k}\left\|\boldsymbol{\theta}_{k}\right\|_{2}\right]
$$

where $\boldsymbol{\theta}_{k}$ is the set of RPs in group $k$ and $w_{k}$ is their corresponding weights, and $\mathbf{y}=\boldsymbol{\Phi} \boldsymbol{y}$ is the AP selected online measurements. The structure of matrix $\boldsymbol{\Phi}$ is defined in Section V. A new method for defining the groups and their corresponding weights is proposed in Section IV. The first element of (2) reduces the possible impact of the online fingerprint noise and outliers by minimizing the residuals in the linear equation system and finds the best user's position. The second component searches for a sparse solution over all possible RPs so that only a small subset of them are selected. The last term provides the sparsity over the clusters so that the recovered vector nonzero elements concentrates in groups with higher weights. This term basically plays the role of coarse localization in the literature. The formulation in (2) is known as Sparse Group Lasso (SGL) and is applied for the first time in this paper in the context of WLAN positioning [13]. Customized solvers are also available; see e.g. [14].

As mentioned earlier, typical localization schemes rely on coarse localization preprocessing to reduce the size of the problem to search over a smaller region of area. However, if a wrong subset of RPs is selected, the localization error becomes very large. Therefore, the advantage of our proposed group sparsity approach is that the coarse localization scheme is embedded in the fine localization formulation.

\section{ONLINE LAYERED CLUSTERING}

In this section, we propose a new method to define the groups of RPs and their corresponding weights. The RPs are divided into layered groups while each group contributes to localization based on the weights assigned to that group. First, let define the AP coverage vector for the radio map as $\mathbf{I}_{j}=\left[I_{j}^{1}, \ldots, I_{j}^{L}\right]$, where $I_{j}^{i}=1$ if AP $i$ provides continuous coverage at $\mathbf{p}_{j}$ and is 0 otherwise. An AP provides continuous coverage at $\mathbf{p}_{j}$ if its fingerprints are above a threshold $\gamma$ for 90 percent of the time [2]. Similarly, for online vector $\boldsymbol{y}$, the coverage vector $\mathbf{I}_{\boldsymbol{y}}$ has its $i$-th entry set to 1 if the online measurement from AP $i$ is above $\gamma$, and zero otherwise. The 


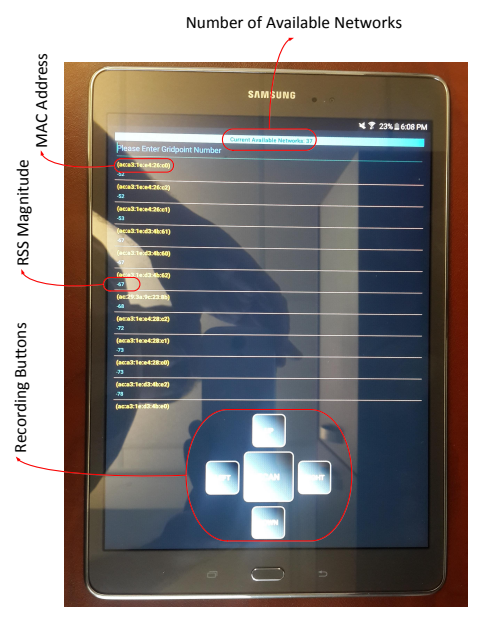

Fig. 1. The fingerprinting device with the developed Android application.

Hamming distance between two binary vectors $\mathbf{I}_{\boldsymbol{y}}$ and $\mathbf{I}_{j}$ is used to indicate the number of APs with different coverage

$$
d_{H}\left(\mathbf{I}_{\boldsymbol{y}}, \mathbf{I}_{\mathbf{p}_{j}}\right)=\sum_{i=1}^{L}\left|I_{y}^{i}-I_{j}^{i}\right| \quad \forall j \in\{1, \ldots, N\} .
$$

For online layered clustering, at first, the distance between the online measurement coverage vector and that of each RP is computed using (3). We define the minimum and maximum of the Hamming distance over the area as

$$
\begin{aligned}
d_{H}^{\min } & =\min _{j=1, \ldots, N} d_{H}\left(\mathbf{I}_{\boldsymbol{y}}, \mathbf{I}_{j}\right) \\
d_{H}^{\max } & =\max _{j=1, \ldots, N} d_{H}\left(\mathbf{I}_{\boldsymbol{y}}, \mathbf{I}_{j}\right) .
\end{aligned}
$$

Then, the Hamming difference range over the whole area and the group Hamming range is defined, respectively, as following

$$
\begin{aligned}
R_{H} & =d_{H}^{\max }-d_{H}^{\min } \\
r & =\frac{R_{H}}{K}
\end{aligned}
$$

where $K$ is the number of groups (clusters). RPs are clustered with respect to their Hamming distances to the online measurement. Specifically, the distance range $\left[d_{H}^{\min }, d_{H}^{\max }\right]$ is partitioned in $K$ groups collected in set $\mathcal{D}$

$$
\mathcal{D}=\left\{\left(d_{k-1}, d_{k}\right) \mid d_{k}=d_{H}^{\text {min }}+k r, k=0, \ldots, K\right\} .
$$

Then, $\mathbf{p}_{j}$ is assigned to group $k$ if and only if

$$
d_{k-1} \leq d_{H}\left(\mathbf{I}_{\boldsymbol{y}}, \mathbf{I}_{j}\right) \leq d_{k} \quad \forall k=1, \ldots, K .
$$

Note that $\mathbf{p}_{j}$ cannot belong to more than one group. The corresponding weight for each group is the inverse of the average of group Hamming distance

$$
w_{k}=\frac{2}{d_{k-1}+d_{k}} \quad \forall k=1, \ldots, K .
$$

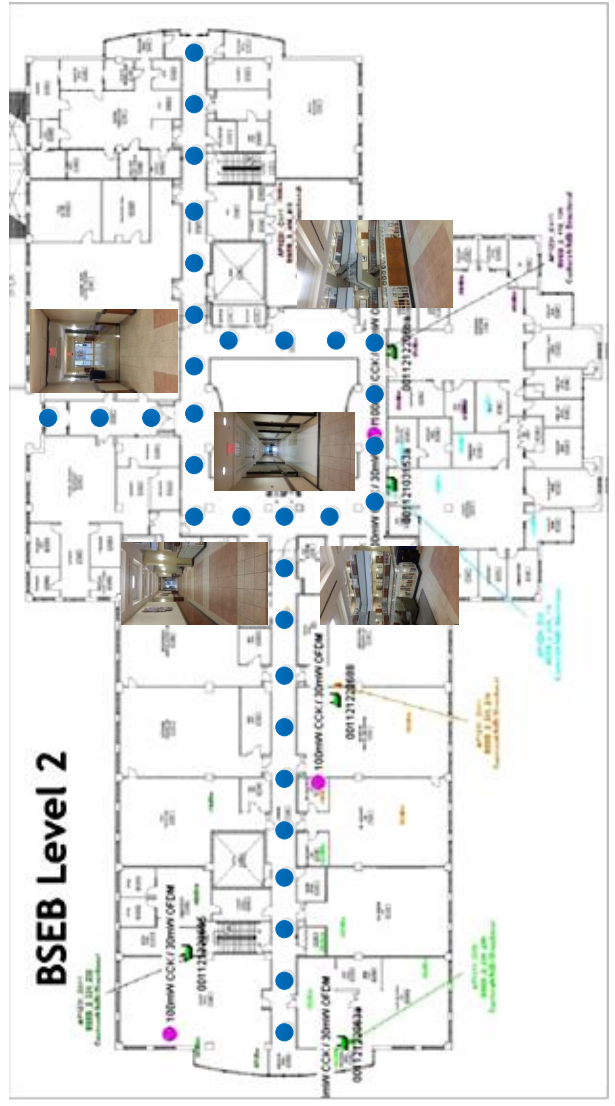

Fig. 2. The real map of the experimenting area.

\section{Selection of APs}

The selection of APs is based on assigning a score to each AP through Fisher criterion. This criterion uses the history of radio map fingerprints and assigns a score to each $\mathrm{AP}$

$$
\zeta^{i}=\frac{\overbrace{\sum_{j=1}^{N}\left(\psi_{j}^{i}-\bar{\psi}^{i}\right)^{2}}^{\frac{1}{M} \sum_{m=1}^{M} \sum_{j=1}^{N}\left(r_{j}^{i}\left(t_{m}\right)-\psi_{j}^{i}\right)^{2}}}{\underbrace{\text { the differentiability of AP } i \text { across RPs }}_{\text {the variance of readings for AP } i}}, i=1, \ldots, L,
$$

The AP selection procedure is modeled by a matrix $\Phi$ whose $i$-th row, $\boldsymbol{\Phi}^{i}$, defines the AP that is selected:

$$
\boldsymbol{\Phi}^{i}=[0, \cdots, \underbrace{1}_{\text {Index of selected AP }}, \cdots, 0] \forall i=1, \cdots,|\mathcal{L}|, \mathcal{L} \subset \mathcal{A}
$$

\section{RESUlTS AND Discussion}

For verifying the accuracy and granularity of the proposed approach, we have evaluated the GS-based localization method 


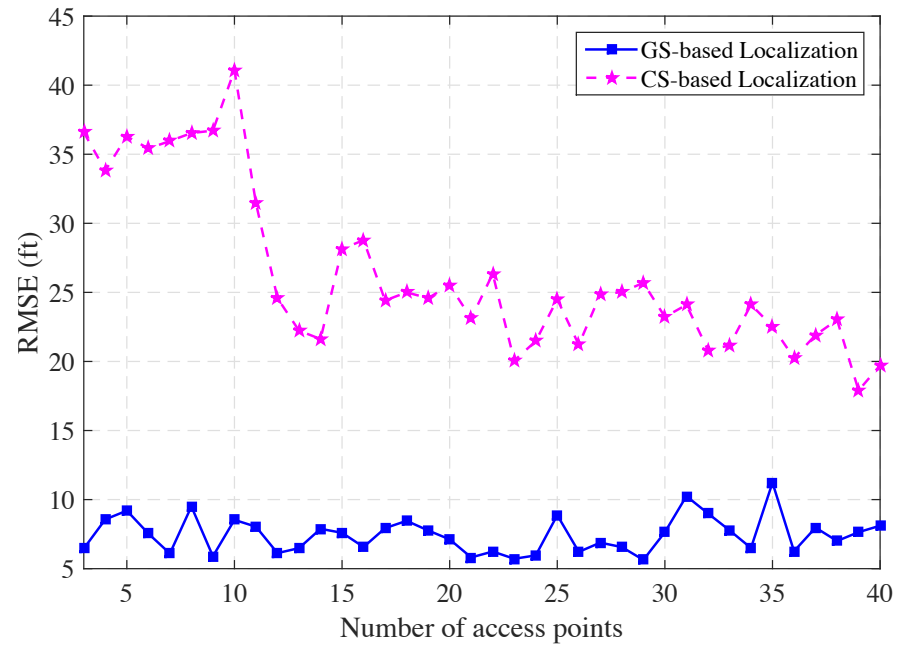

Fig. 3. The average RMSE of the estimated positions for CS-based and GSbased localization for different number of APs. The results show that the localization error has the least error when 9 APs are used for localization.

in a real environment. A collection of fingerprints have been recorded using a Samsung Tablet (Galaxy Tab A) and a self-developed Android application functioning on Android Lollipop 5.0.2 and utilizing the inherent android.net.wifi package features. Fig. 1 shows a picture of the application. The application reads the Network Interface Card (NIC) once per second to ensure obtaining the updated RSS values and also records the media access control (MAC) address of each AP along with its RSS magnitudes.

The fingerprints have been collected from the second floor of the Biotechnology Sciences and Engineering (BSE) Building at the University of Texas at San Antonio which has area of $114 \mathrm{ft} \times 347 \mathrm{ft}$. The building features a representative indoor office environment with complex wireless propagation patterns due to research labs, offices, café terria, and study areas, and has a high commuting volume. The area has been divided into 182 RPs and a collection of 100 samples per RP has been recorded over two months. Although existing methods design their settings with 5-9 $\mathrm{ft}$ spacing, we found $3 \mathrm{ft}$ to be a sound grid spacing. A total of 1238 different MAC addresses were visible. Fig. 2 shows the map of the area where the blue dots indicate the fingerprinting locations.

The evaluation criteria for the estimated positions is computed as the average Euclidean distance between the estimated and the true locations of the random points

$$
\operatorname{RMSE}=\frac{1}{N_{t}} \sum_{j=1}^{N_{t}} \sqrt{(\hat{\mathbf{p}}(j)-\mathbf{p}(j))^{T}(\hat{\mathbf{p}}(j)-\mathbf{p}(j))}
$$

known as Root Mean Square Error (RMSE) where $N_{t}$ is the number of test points. For better evaluation of the distribution of the errors, the cumulative distribution function (CDF) of the errors has also been investigated.

Fig. 3 shows the RMSE for CS-based and GS-based (10 groups, $\lambda_{1} / \lambda_{2}=0.1$ ) when the number of APs included in positioning is increased. The performance of CS-based

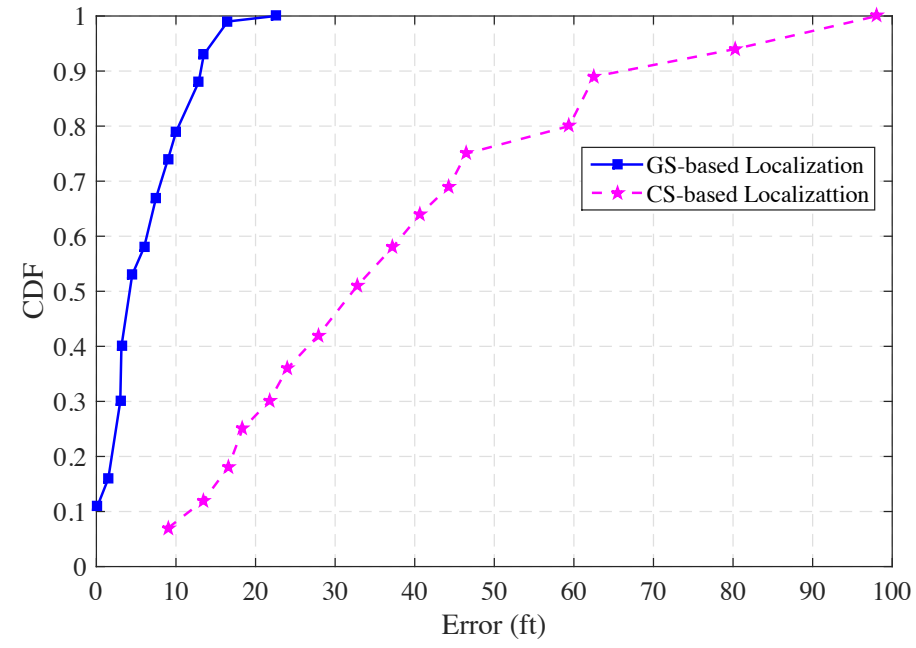

Fig. 4. The cumulative position error for GS-based Localization compared to CS-based approach. Most of the errors are concentrated below $10 \mathrm{ft}$.

localization degrades if the number of observations is small. However, for GS-based localization when the number of APs is increasing from 3 to 9 , the localization error decreases as introducing more online measurement leads to a better sparse user's location recovery. However, the results show that increasing the number of APs does not necessarily increase the positioning accuracy as the localization error is the least when 9 APs are used. The error increases afterwards as the as introducing more APs may lead to biased estimations.

Another feature that should be taken into account is the distribution of the errors. To this end, we have observed the distribution of the errors when 9 APs are being used. Fig. 4 shows that CS-based localization suffers from large localization errors. The proposed GS-based localization shows very desired CDF characteristics as most of the errors are concentrated below $10 \mathrm{ft}$ with the maximum at $22 \mathrm{ft}$.

\section{CONCLUSIONS}

In this paper, we proposed a novel indoor WLAN localization scheme which does not need the pre-processing offline clustering and sbsequent online coarse localization. Instead, the are is divided into groups through a comparison metric between the online and radio map fingerprints. All of these groups are engaged in localization through their corresponding distance. The simulation results show that the proposed method has a high localization accuracy and promising choice for real localization applications.

\section{REFERENCES}

[1] M. Youssef, A. Agrawala, and A. Udaya Shankar, "WLAN location determination via clustering and probability distributions," in Proceedings of the First IEEE International Conference on Pervasive Computing and Communications, March 2003, pp. 143-150.

[2] A. Kushki, K. Plataniotis, and A. Venetsanopoulos, "Kernel-based positioning in wireless local area networks," IEEE Transactions on Mobile Computing, vol. 6, no. 6, pp. 689-705, June 2007. 
[3] Y. Chen, Q. Yang, J. Yin, and X. Chai, "Power-efficient accesspoint selection for indoor location estimation," IEEE Transactions on Knowledge and Data Engineering, vol. 18, no. 7, pp. 877-888, July 2006.

[4] A. Kushki, K. Plataniotis, A. Venetsanopoulos, and C. Regazzoni, "Radio map fusion for indoor positioning in wireless local area networks," in 8th International Conference on Information Fusion, vol. 2, July 2005.

[5] A. Tabibiazar and O. Basir, "Compressive sensing indoor localization," in IEEE International Conference on Systems, Man, and Cybernetics (SMC), Oct 2011, pp. 1986-1991.

[6] C. Feng, W. Au, S. Valaee, and Z. Tan, "Received-signal-strength-based indoor positioning using compressive sensing," IEEE Transactions on Mobile Computing, vol. 11, no. 12, pp. 1983-1993, Dec 2012.

[7] J. Deng, Q. Cui, X. Zhang, and X. Xu, "Compressive sensing based indoor positioning with denosing and filtering in LF space," in $23 \mathrm{rd}$ International Symposium on Personal Indoor and Mobile Radio Communications (PIMRC), Sept 2012, pp. 2477-2482.

[8] S.-H. Fang, T.-N. Lin, and K.-C. Lee, "A novel algorithm for multipath fingerprinting in indoor WLAN environments," IEEE Transactions on Wireless Communications, vol. 7, no. 9, pp. 3579-3588, September 2008.

[9] E. Kupershtein, M. Wax, and I. Cohen, "Single-site emitter localization via multipath fingerprinting," IEEE Transactions on Signal Processing, vol. 61, no. 1, pp. 10-21, Jan 2013.

[10] K. Kaemarungsi and P. Krishnamurthy, "Modeling of indoor positioning systems based on location fingerprinting," in Twenty-third Annual Joint Conference of the IEEE Computer and Communications Societies, vol. 2, March 2004, pp. 1012-1022 vol.2.

[11] P. Bahl and V. N. Padmanabhan, "RADAR: An in-building RF-based user location and tracking system," in IEEE International Conference on Computer Communications, 2000, pp. 775-784.

[12] T. Roos, P. Myllymäki, H. Tirri, P. Misikangas, and J. Sievänen, "A probabilistic approach to WLAN user location estimation." International Journal of Wireless Information Networks, vol. 9, no. 3, pp. 155-164, 2002.

[13] J. Liu, S. Ji, and J. Ye, SLEP: Sparse Learning with Efficient Projections, Arizona State University, 2009.

[14] N. Simon, J. Friedman, T. Hastie, and R. Tibshirani, "A sparse-group lasso," Journal of Computational and Graphical Statistics, 2013. 